\title{
Nutrient transport suggests an evolutionary basis for charged archaeal surface layer proteins
}

\author{
Po-Nan Li (i) ${ }^{1,2}$ - Jonathan Herrmann ${ }^{2,3}$ - Bradley B. Tolar (1) ${ }^{4}$. Frédéric Poitevin ${ }^{3,5}$ - Rasika Ramdasi ${ }^{6}$ • \\ John R. Bargar ${ }^{2}$. David A. Stahl ${ }^{7}$. Grant J. Jensen ${ }^{6,8}$. Christopher A. Francis ${ }^{4}$ - Soichi Wakatsuki ${ }^{2,3}$. \\ Henry van den Bedem $\mathbb{D}^{2,9}$
}

Received: 30 November 2017 / Revised: 11 April 2018 / Accepted: 14 April 2018 / Published online: 13 June 2018

(c) International Society for Microbial Ecology 2018

\begin{abstract}
Surface layers (S-layers) are two-dimensional, proteinaceous, porous lattices that form the outermost cell envelope component of virtually all archaea and many bacteria. Despite exceptional sequence diversity, S-layer proteins (SLPs) share important characteristics such as their ability to form crystalline sheets punctuated with nano-scale pores, and their propensity for charged amino acids, leading to acidic or basic isoelectric points. However, the precise function of S-layers, or the role of charged SLPs and how they relate to cellular metabolism is unknown. Nano-scale lattices affect the diffusion behavior of low-concentration solutes, even if they are significantly smaller than the pore size. Here, we offer a rationale for charged S-layer proteins in the context of the structural evolution of S-layers. Using the ammonia-oxidizing archaea (AOA) as a model for S-layer geometry, and a 2D electrodiffusion reaction computational framework to simulate diffusion and consumption of the charged solute ammonium $\left(\mathrm{NH}_{4}{ }^{+}\right)$, we find that the characteristic length scales of nanoporous S-layers elevate the concentration of $\mathrm{NH}_{4}{ }^{+}$in the pseudo-periplasmic space. Our simulations suggest an evolutionary, mechanistic basis for S-layer charge and shed light on the unique ability of some AOA to oxidize ammonia in environments with nanomolar $\mathrm{NH}_{4}^{+}$availability, with broad implications for comparisons of ecologically distinct populations.
\end{abstract}

\section{Introduction}

Surface layers (S-layers) are proteinaceous macromolecular assemblies that form a two-dimensional (2D) crystal lattice

Electronic supplementary material The online version of this article (https://doi.org/10.1038/s41396-018-0191-0) contains supplementary material, which is available to authorized users.

$\triangle$ Christopher A. Francis

caf@stanford.edu

$\triangle$ Soichi Wakatsuki

soichi.wakatsuki@stanford.edu

$\triangle$ Henry van den Bedem

vdbedem@stanford.edu

1 Department of Electrical Engineering, Stanford University, Stanford, CA 94305, USA

2 SLAC National Accelerator Laboratory, Menlo Park, CA 94025, USA

3 Department of Structural Biology, Stanford University, Stanford, CA 94305, USA on the outside of most archaea and many bacteria [1-3]. Slayer proteins (SLPs) are generally $40-200 \mathrm{kDa}$ and represent up to $15 \%$ of total cellular protein production [4]. S-layers serve as the first selective barrier between the cell and its environment, which includes interactions with predators, symbionts, proteins, nutrients, and energy sources [5]. Bacterial S-layers have been linked to diverse functions, including protection from large macromolecules and

4 Department of Earth System Science, Stanford University, Stanford, CA 94305, USA

5 Stanford PULSE Institute, SLAC National Accelerator Laboratory, Menlo Park, CA 94025, USA

6 Division of Biology and Biological Engineering, California Institute of Technology, Pasadena, CA 91125, USA

7 Department of Civil and Environmental Engineering, University of Washington, Seattle, WA 98195, USA

8 Howard Hughes Medical Institute, California Institute of Technology, Pasadena, CA 91125, USA

9 Department of Bioengineering and Therapeutic Sciences, University of California, San Francisco, CA 94158, USA 
predators, biofilm formation, adhesion, and pathogenesis [6]. Although archaeal S-layers have been implicated in cell shape determination, protection from osmotic shock, and providing a scaffold for biomineralization [7-9], the genetic intractability of most archaea has hindered in-depth physiological study of archaeal S-layer functions.

Molecular sieving by S-layers has been demonstrated in vitro with pore sizes up to $4.5 \mathrm{~nm}$ in width [10]. The molecular architecture of a $2 \mathrm{D}$ crystalline protein barrier with nano-scale pores necessarily affects the diffusion behavior of low-concentration solutes even if they are significantly smaller than the pore size [11-13]. Nonetheless, many archaea thrive in implausibly nutrient-poor environments. Accordingly, adaptation to chronic energy stress has been hypothesized to be the primary factor distinguishing archaea from their bacterial counterparts [14]. Therefore, we reasoned that archaea may have evolved mechanisms to selectively enhance nutrient acquisition using the nanoporous S-layer. To address this question, we analyzed the cellular envelope of ammonia-oxidizing archaea (AOA), ubiquitous microorganisms whose sole energy source is commonly found in low nanomolar concentrations.

Thaumarchaeal [15] AOA are among the most ubiquitous and abundant organisms on Earth [16, 17]. AOA perform the first step of nitrification, oxidizing ammonia $\left(\mathrm{NH}_{3}\right)$ to nitrite $\left(\mathrm{NO}_{2}\right)$, and play a critical role in the global nitrogen cycle [17-19]. AOA often outnumber their bacterial counterparts (ammonia-oxidizing bacteria, AOB) in marine or terrestrial environments, especially under conditions where nutrients are extremely limited [20,21]. AOA have a remarkably high affinity for their substrate. For example, cultures of Nitrosopumilus maritimus strain SCM1 showed exponential growth at substrate concentrations (measured as $\mathrm{NH}_{3}+\mathrm{NH}_{4}{ }^{+}$) below $10 \mathrm{nM}$, and have a half-saturation constant $k_{\mathrm{m}}=133 \mathrm{nM}$ [22]. Similar findings were reported from in situ nitrification measurements [23], and for other AOA [24]. In contrast, $k_{\mathrm{m}}$ values of characterized AOB are at least one to two orders of magnitude higher $[25,26]$. AOB cultures often require concentrations in the micromolar to millimolar range to sustain growth, signifying that AOA have evolved competitive advantages in environments where AOB cannot survive. However, the macromolecular and cellular adaptations that allow AOA to occupy these ecological niches remain unresolved.

The AOA obtain energy directly from electrons evolved during ammonia oxidation [19]. This process is attributed to the ammonia monooxygenase (AMO) metallo-enzyme complex and a copper-based electron transport system [19]. AMO is believed to be a heterotrimeric, membraneassociated complex with each protomer composed of three subunits: AmoA, AmoB, and AmoC [27]. Although the specific location of the archaeal AMO active site is unknown, the bacterial homolog indicates a location within the AmoB subunit, which is mostly soluble and resides underneath the S-layer in the pseudo-periplasmic space (PPS) [28-30]. However, archaeal AmoB sequences are shorter $(20 \mathrm{kDa})$ and align only with the first half of bacterial AmoB. The second half of bacterial AmoB is missing from all known AOA genomes and metagenomes, suggesting that the archaeal AMO may catalyze ammonia oxidation by a different, uncharacterized mechanism [30, 31], possibly involving $\mathrm{NH}_{4}{ }^{+}$directly. Although $\mathrm{NH}_{3}$ was suggested as substrate for AOB [32], the substrate for archaeal AMO, $\mathrm{NH}_{3}$ or $\mathrm{NH}_{4}^{+}$, is not firmly established [29, 33].

Precisely how a charged S-layer and nanoporous lattice affect availability of nutrients in the PPS is not known. The cellular surface of $N$. maritimus, a model AOA, is enriched in reactive sites, consistent with charged amino acids such as aspartic and/or glutamic acid, compared with AOB [34]. The electric double layer (EDL) resulting from charged and reactive surface amino acids attracting positively charged ammonium ions could act as a nutrient reservoir [34]. Further, synthetic nanochannels lined with surface charges have been shown to enter a 'constant-conductance' regime at low ionic strengths [35-38]. Below micromolar concentrations, channel conductance is near constant, governed by the surface charges independent of concentration, and higher than predicted from bulk behavior, thus controlling ion transport.

Here, we quantitatively model the cellular envelope of the AOA to explore mechanisms underpinning their extraordinarily high affinity for ammonium. We formulated a 2D Poisson-Nernst-Planck (PNP) electrodiffusion reaction transport model to probe interactions between the environment, the S-layer, a pore, AMO, its substrate, and anions (Materials and methods section). Our non-equilibrium simulations, informed by electron cryotomographic imaging, suggest that a charged S-layer can double the $\mathrm{NH}_{4}{ }^{+}$ concentration at a theoretical pseudo-periplasmic active site at low environmental concentrations, elevating the apparent $k_{\mathrm{m}}$ of the enzyme's substrate. The effect is not large enough to explain the difference between AOA and AOB (10 vs. $1000 \mathrm{nM}$ ), suggesting additional molecular adaptations. However, we found that a charged S-layer surface mitigates diffusion limitations imposed by a physical permeability barrier. As a result, we elucidate a possible functional and evolutionary basis for charged S-layers and highlight their ecological importance to AOA.

\section{Results}

\section{Sequence diversity and charge conservation in archaeal SLPs}

Amino-acid sequence analysis of 25 putative SLPs (Table S1) revealed very low sequence conservation 

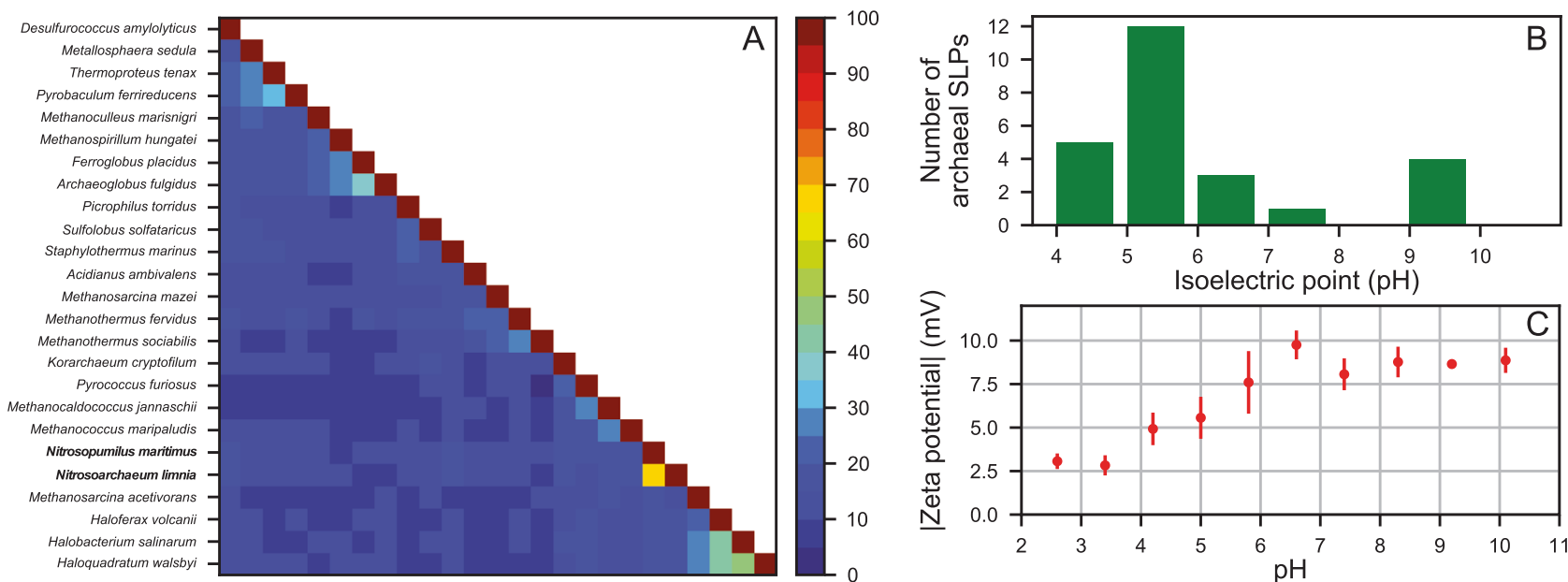

Fig. 1 SLPs share a propensity for charged amino acids, despite exceptional sequence diversity. a Amino-acid sequence identity matrix for putative S-layer proteins from all known archaeal clades. Color codes indicate the \% identity. b Isoelectric points for the SLPs analyzed in a show clusters of sequences with notable negative or positive

between archaeal clades (Fig. 1a). Strikingly, despite their genomic diversity, the majority of sequences maintain a theoretical isoelectric point far from neutral (Fig. 1b). Of the 25 SLP sequences shown, 21 are predicted to be negatively charged and 4 positively charged, with a clear dearth of sequences in the neutral regime (Fig. 1b).

The amino-acid sequence of Nmar1201, one of two large, homologous SLPs in the N. maritimus SCM1 genome, has a theoretical isoelectric point of 3.5, the 9th lowest in its entire genome. To confirm that theoretical isoelectric point is a good indicator for a charged archaeal surface in vivo, we measured the Zeta potential of an AOA closely related to N. maritimus, Nitrosoarchaeum limnia [39], at $\mathrm{pH}$ values between 2 and 10 (Fig. 1c). These data indicate $\mathrm{pH}$ sensitive surface charge with a Zeta potential around -9 $\mathrm{mV}$ at $\mathrm{pH}$ values above 6 but $-3 \mathrm{mV}$ at highly acidic $\mathrm{pH}$. The switch between high and low charge states occurs between $\mathrm{pH} 4$ and $\mathrm{pH} 6$ (Fig. 1c). The N. limnia SLP, Nlim1951, exhibits similar length and 65\% sequence identity with its homologous sequence in $N$. maritimus, Nmar1201. Indeed, the predicted isoelectric points for Nlim1951 and Nmar1201 are 4.08 and 3.77, respectively. Although we would predict a global Zeta potential minimum at the S-layer isoelectric point of $\mathrm{pH} 4$, we see constant values at lower $\mathrm{pH}$ (Fig. 1c). This may be due to acidinduced S-layer shedding, which has been observed in bacterial systems [40]. This phenomenon can be attributed to modulation of hydrogen bonding within the S-layer lattice, which may disrupt anchoring, crystallization contacts, or overall stability of the protein fold. Although SLPs are model proteins for N-linked glycosylation in halophilic or thermophilic archaea [41, 42], analysis of protein charge at neutral $\mathrm{pH}$. $\mathbf{c}$ Zeta potential measurements of living $N$. limnia SFB1 cells in growth medium plotted as a function of $\mathrm{pH}$. The absolute value of the potential is reported. Error bars show the length of one standard deviation

glycosylation by mass spectrometry of whole-cell lysates of $N$. limnia indicated no evidence for $\mathrm{N}$-glycans (Suppl. Methods). This finding is further supported by our Zeta potential experiments: glycosylation would affect the Slayer surface charge, but measured Zeta potentials agree with the predicted isoelectric point of unmodified SLPs. On the basis of theoretical isoelectric points of 25 archaeal SLPs and Zeta potential measurements of AOA cells, we reason that SLPs create charged outer surfaces on archaea and that this property is evolutionarily conserved.

\section{Molecular organization of the AOA cellular envelope}

To characterize the molecular organization of AOA SLPs and AMO, we collected electron cryo-tomograms of N. maritimus (Fig. 2a). The tomograms revealed SLPs arranged in a hexagonal pattern covering the entire cell. Hexagonal symmetry is a predominant feature of archaeal S-layers [2, 43]. We then averaged 200 S-layer subunits to obtain detailed images of the subunits at $4 \mathrm{~nm}$ resolution (Fig. 2b). The averaged volume clearly shows six subunits within each structural unit. The center-to-center distance between two hexagonal S-layer subunits is $22 \mathrm{~nm}$ and the distance between two neighboring vertices of the hexagons is $8 \mathrm{~nm}$. Each hexagonal structural unit consists of a central nanopore positioned above a central, membrane-proximal electron-dense structure (Figs. 2c, d).

Crystal structures of AOA SLPs and the AMO heterotrimer have remained elusive. Proteomic analysis of N. maritimus indicated two types of SLPs: one large (180 $\mathrm{kDa})$ and one small $(25 \mathrm{kDa})$ [44]. Genome analysis indicates multiple homologous copies of each subunit: two of 
Fig. 2 SLPs create charged, nanoporous outer surfaces on archaea. a Slice through a 3-D electron cryotomographic image of an N. maritimus cell showing S-layer subunits. The hexagonal pattern of the S-layer is visible at magnification of $\times 22,000$.

b, c Subtomogram average of S-layer subunits, showing individual hexagonal S-layer subunits: top slices show a central pore (b) and bottom slices show electron-dense Slayer structures, possibly a complex associated with AMO (c). d A side view of the tomograms reveals the interior arrangement of the periodic structures. e Molecular organization of S-layer proteins and AMO. S-layer proteins are anchored in the membrane at the $\mathrm{N}$-terminus, forming a canopy to enclose a PPS. Trimers of AMO heterotrimers are anchored in the membrane, with active sites exposed to PPS. Nutrients need to pass through pores in the S-layer
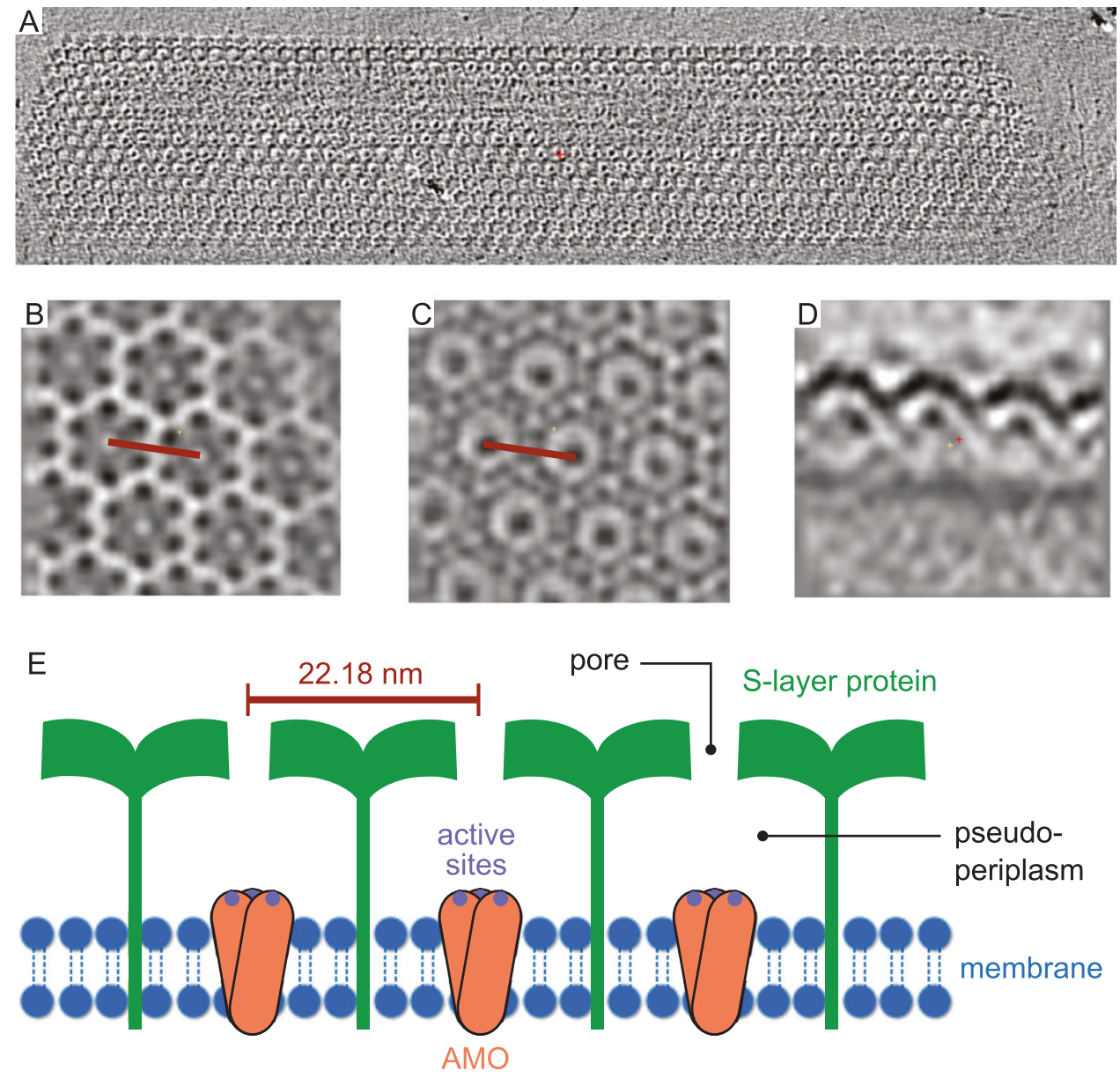

the large subunit (Nmar1201 and 1547) and four of the small subunit (Nmar0343, 0344, 0345, and 1257). Sequence analysis of the $180 \mathrm{kDa}$ SLPs suggested a 20-residue hydrophobic helix at the N-terminus [45, 46], which likely plays a role in anchoring the protein in the membrane, either directly [43] or by serving as a signaling peptide for lipid modification [47]. Although structures are not available for the AmoA or AmoC subunits of AMO, there is considerable evidence that they are integral membrane proteins [30]. The central electron-dense features observed in the tomograms would readily accommodate AMO. Based on AMO sequences, the molecular mass of the AMO trimer of heterotrimers would be approximately $300 \mathrm{kDa}$. However, most of the mass is contained within the membrane. The archaeal (soluble) AmoB subunit of Nitrosocaldus yellowstonii is $20 \mathrm{kDa}$ and $4.5 \mathrm{~nm}$ in size, suggesting that additional proteins would likely occupy the electron-dense area. These observations, together with the cryoelectron tomograms, motivate a model of the molecular organization of ammonia oxidation in which active sites of AMO are exposed to a PPS formed by SLPs (Fig. 2e). Nutrients, for example, ammonium, must pass through the S-layer nanopores to access the AMO active sites.

\section{An electrodiffusion reaction transport model for the $A O A$ cell envelope}

To examine how a charged S-layer modulates substrate availability in the PPS, we designed a continuum electrodiffusion reaction transport model. We represent a single hexagonal unit cell of the S-layer by a 2D, rectangular, periodic simulation domain (Fig. 3, Materials and methods).

The S-layer was modeled as a $4.5 \mathrm{~nm}$ thick, impenetrable boundary with fixed surface charges. The pseudoperiplasmic domain measures $18 \times 22 \mathrm{~nm}$, and is connected to the extracellular domain by a single nanopore also lined with surface charges, $1.3 \mathrm{~nm}$ in diameter (d) (Fig. 3a). The bulk solution is composed of three ion species, ammonium $\left(\mathrm{NH}_{4}{ }^{+}\right)$with a positive unit charge, a sodium $\left(\mathrm{Na}^{+}\right)$co-ion, and a chloride counterion $\left(\mathrm{Cl}^{-}\right)$with a negative unit charge to maintain electroneutrality at $n_{\infty}$ (bulk). We examined $\mathrm{NH}_{4}{ }^{+}$bulk concentrations of 10,100 , and $1000 \mathrm{nM}$, fixed $\mathrm{Na}^{+}$and $\mathrm{Cl}^{-}$concentrations of $0.14 \mathrm{M}$ and fixed $\mathrm{S}$-layer surface charges equivalent to a potential of $-140 \mathrm{mV}$ in the Gouy-Chapman model of charge distribution (Materials and methods). These parameters correspond to conditions frequently encountered by marine AOA 

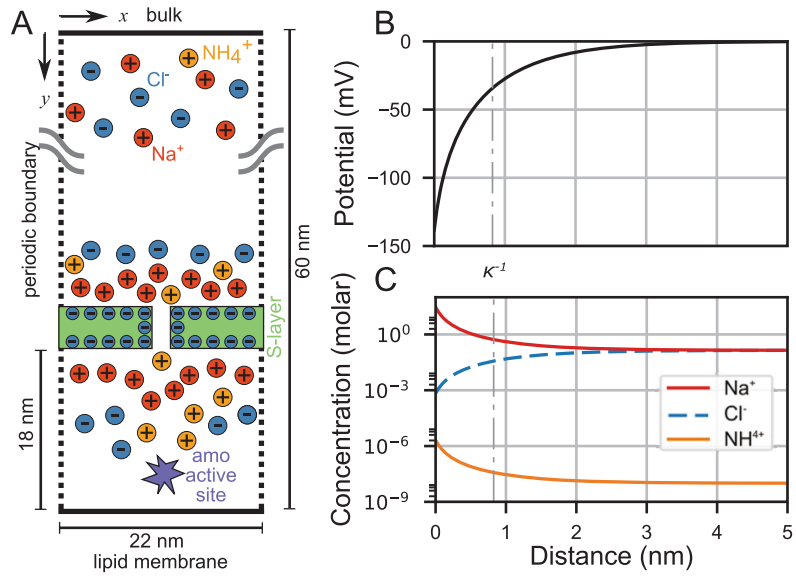

Fig. 3 Simulation domain and equilibrium characteristics. a A periodic, 2D $22 \times 60 \mathrm{~nm}$ simulation domain representing a hexagonal cell of the S-layer. The bottom of the S-layer is $18 \mathrm{~nm}$ from the lipid membrane. The boundary facing the lipid membrane (bottom) is considered impenetrable, represented by a zero-flux Neumann boundary condition. The upper (bulk) boundary is represented by a diffusive boundary condition, allowing ions to diffuse into the simulation domain. b Electric potential and (c) ion concentrations as a function of distance from the S-layer extracellular surface, calculated for $10 \mathrm{nM} \mathrm{NH}{ }_{4}^{+}$bulk concentrations using the Poisson-Boltzmann equations at equilibrium (no sink) and an S-layer without pore. $\mathrm{Na}^{+}$ and $\mathrm{Cl}^{-}$concentrations are $0.14 \mathrm{M}$. The Debye length scale $\kappa^{-1}$ equals $0.82 \mathrm{~nm}$, and is largely determined by $\mathrm{Na}^{+}$and $\mathrm{Cl}^{-}$concentrations

[22]. The AMO active site is modeled as a point-like ion sink at a fixed location, $2.5 \mathrm{~nm}$ above the membrane in PPS. From laboratory experiments, we estimated an ammonia oxidation turnover rate of $k=10 s^{-1}$ (Materials and methods).

The high, molar-range co-ion and counterion bulk concentrations lead to extremely short characteristic length scales for the simulated system. We analytically calculated electric potentials, concentration profiles and Debye lengths $\kappa^{-1}$ at equilibrium (Fig. 3b, c, Materials and methods). The Debye length is $0.82 \mathrm{~nm}$, and nearly constant across the 10-1000 nM range of $\mathrm{NH}_{4}{ }^{+}$concentrations, owing to $\mathrm{Na}^{+}$ and $\mathrm{Cl}^{-}$concentrations that are many orders of magnitude higher. We selected a simulation domain at $60 \mathrm{~nm}$ in height, many times larger than $\kappa^{-1}$, and maintained the bulk concentrations at the bulk boundary. Starting from equilibrium profiles, we then numerically solved the PNP equations. The dynamical system converged rapidly to a steady state (Materials and methods, Fig. S1A,B).

\section{A charged S-layer alters pseudo-periplasmic ion concentrations and reaction turnover}

We examined the effect of a neutral and charged S-layer on $\mathrm{NH}_{4}{ }^{+}$concentration profiles in PPS, compared with the absence of an S-layer. Without S-layer, the $\mathrm{NH}_{4}{ }^{+}$concentration in the entire simulation domain was consistently below the bulk concentration, owing to the active site consuming $\mathrm{NH}_{4}{ }^{+}$(Fig. 4a, Table 1). For a neutral S-layer, concentration levels exterior to the S-layer were slightly higher than those found in the absence of an S-layer (Fig. 4b, Table 1). However, pseudo-periplasmic concentrations were nearly fourfold lower than extraplasmic concentrations, signifying that the nanopore restricted diffusion of small molecules at steady state (Fig. 4b, Table 1). Strikingly, a negatively charged S-layer restored $\mathrm{NH}_{4}{ }^{+}$ concentration throughout the simulation domain to levels nearly indistinguishable from those found in the absence of an S-layer, except near the S-layer where concentrations were much higher (Fig. 4c, d, Table 1).

Notably, active site $\mathrm{NH}_{4}{ }^{+}$concentrations with a negatively charged S-layer increased approximately twofold (197\% at 10,100, and $1000 \mathrm{nM}$ ) compared with a neutral Slayer (Fig. 4d, Table 1). The short, nanometer-range Debye lengths limit the effect of the EDL to an immediate neighborhood of the charged S-layer, resulting in strongly peaked concentrations elevated nearly two orders of magnitude from the bulk concentration $(0.340,3.41,34.9 \mu \mathrm{M}$ at 10 , 100, $1000 \mathrm{nM}$, respectively, Fig. 4d). Inside the pore, the $\mathrm{NH}_{4}{ }^{+}$concentration was lower than at the S-layer boundary, but remained elevated three-fold from bulk (Fig. 4d, Table 1). Below the charged S-layer the concentration levels dropped sharply, but the concentration profiles showed near-identical patterns for the scenarios without Slayer, with neutral S-layer, and with charged S-layer (Fig. 4d). These results similarly held for $\mathrm{NH}_{4}{ }^{+}$concentrations ranging from 10 to $1000 \mathrm{nM}$. Under our assumption of first-order Michaelis-Menten kinetics, the reaction turnover is proportional to the concentration of substrate at the active site. Consequently, a negatively charged S-layer can elevate enzymatic turnover up to twofold at low bulk concentration over neutral S-layers.

Simulations with a positively charged S-layer revealed that it acts as a nutrient barrier, dramatically reducing $\mathrm{NH}_{4}{ }^{+}$ concentrations at the active site (Fig S2). Simulations with the neutral substrate $\mathrm{NH}_{3}$ instead of $\mathrm{NH}_{4}{ }^{+}$confirmed that $\mathrm{S}$ layer charge has no effect on $\mathrm{NH}_{3}$ availability at the active site (Fig S3). At $\mathrm{NH}_{3}$ bulk concentration of $0.20 \mathrm{nM}$, corresponding to a total $\mathrm{NH}_{3}+\mathrm{NH}_{4}{ }^{+}$concentration of $10 \mathrm{nM}$ at $\mathrm{pH}=7.5$, active site $\mathrm{NH}_{3}$ concentration plunged to 24 pM at steady state (Fig S3).

\section{Surface charges render the nanopore cation selective}

At ionic strengths where the Debye length and nanopore diameter have similar order of magnitude, that is, $\kappa d \sim 1$, the concentration of mobile cations in the nanopore will be significantly enriched compared with bulk, whereas the concentration of anions will be greatly reduced. Our 

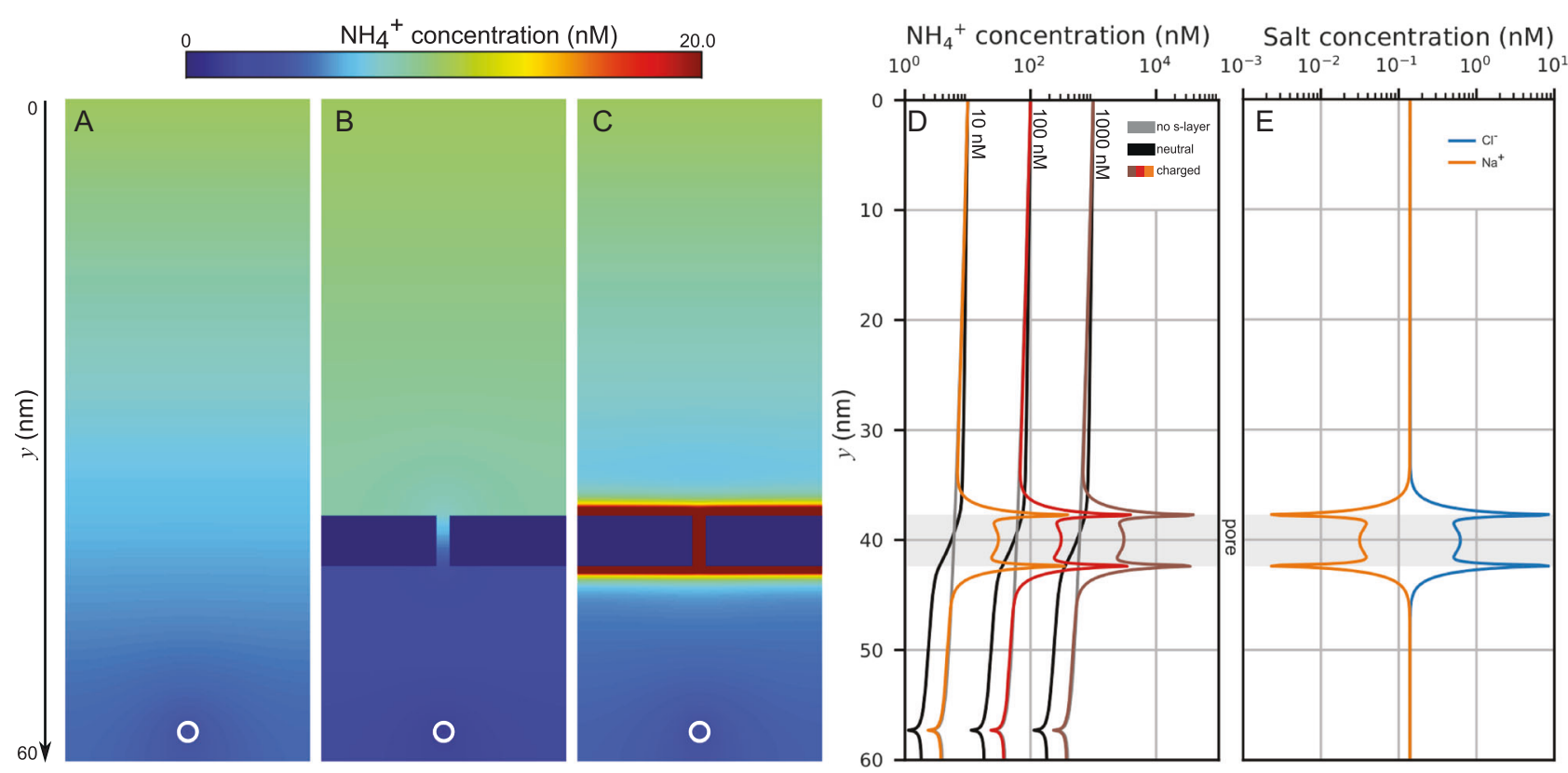

Fig. 4 Charged S-layers control ion concentrations and reaction turnover. a $\mathrm{NH}_{4}{ }^{+}$concentration profile in the simulation domain at steady state in the absence of an S-layer, at a bulk concentration of $10 \mathrm{nM}$. The $\mathrm{NH}_{4}{ }^{+}$gradient is color-coded from low (blue) to high (red) concentration. The position of the point sink (active site) is indicated with a white circle. b $\mathrm{NH}_{4}{ }^{+}$concentration profile with a neutral Slayer. The position and geometry of the S-layer is indicated by dark blue bars. $\mathbf{c} \mathrm{NH}_{4}{ }^{+}$concentration profile with a charged S-layer. $\mathbf{d ~ N H}$
+ concentration profiles for a charged S-layer across the length of the simulation domain at the $x$-coordinate of the point sink, at bulk concentrations of 10 (orange), 100 (red), and 1000 (brown) nM. The corresponding profiles without S-layer and with a neutral S-layer are shown in gray and black. The position of the nanopore is indicated with a gray band. $\mathrm{e} \mathrm{Na}^{+}$and $\mathrm{Cl}^{-}$concentration profiles for a charged S-layer across the length of the simulation domain
Table $1 \mathrm{NH}_{4}^{+}$concentration $[\mathrm{nM}]$ throughout the simulation domain

\begin{tabular}{lllll}
\hline S-layer & $\begin{array}{l}\text { Outside cell } \\
(y=30 \mathrm{~nm})\end{array}$ & $\begin{array}{l}\text { In pore } \\
(y=40 \\
\mathrm{nm})\end{array}$ & $\begin{array}{l}\text { Pseudo } \\
\text { periplasm } \\
(y=50 \mathrm{~nm})\end{array}$ & $\begin{array}{l}\text { Reaction site } \\
(y=57.4 \mathrm{~nm})\end{array}$ \\
\hline None & 7.04 & 6.05 & 5.01 & 2.48 \\
Neutral & 8.52 & 5.61 & 2.47 & 1.20 \\
Charged & 7.12 & 26.6 & 4.82 & 2.37 \\
\hline
\end{tabular}

simulations indicate that at steady state, the concentration of $\mathrm{Na}^{+}$in the nanopore is one to two orders of magnitude larger than that of $\mathrm{Cl}^{-}$(Fig. 4e).

To examine how surface charge controlled ion transport in the nanopore, we measured the current carried by each of the ion species $\mathrm{NH}_{4}^{+}, \mathrm{Na}^{+}$, and $\mathrm{Cl}^{-}$at the pore (Fig. 5a, b; Table S2). For the neutral S-layer, $\mathrm{NH}_{4}{ }^{+}$carried a current of $\mathrm{I}_{\mathrm{NH} 4+}=-1.7 \mathrm{e}-20 \mathrm{~A}$ at steady state at $10 \mathrm{nM}$ concentration. The current is negative owing to an electric field oriented outward of PPS (Fig. 5c, Materials and methods). Our simulations indicated that co- and counterions also carried a small current with a neutral S-layer (Fig. 5c; Fig S4A; Table S2). As a result, we observed an excess of co-ions and lack of counterions around the point-like ion sink location at steady state (Supplementary Note). For a charged S-layer, the $\mathrm{NH}_{4}{ }^{+}$current doubled to $\mathrm{I}_{\mathrm{NH} 4+}=3.3 \mathrm{e}-20 \mathrm{~A}, \mathrm{Na}^{+}$carried a current of $-1.6 \mathrm{e}-20 \mathrm{~A}$, and $\mathrm{Cl}^{-}$of $-1.6 \mathrm{e}-20 \mathrm{~A}$ (Fig. 5b, c; Fig S4B). The parabolic $\mathrm{NH}_{4}{ }^{+}$current profile of the charged nanopore is consistent with an electrophoresisdominated ionic transport model, that is, slip of the EDL (Fig. 5d). By contrast, the current profile of the neutral pore is flat. Interestingly, polarity of the nanopore inverted from minimally positive $(6.7 \mathrm{pV})$ on the PPS side for a neutral Slayer, to $-24 \mathrm{pV}$ for a charged S-layer (Fig. 5c, Materials and methods, Table S2). $\mathrm{NH}_{4}{ }^{+}$conductance of the pore inverted from $\mathrm{G}=-2.5 \mathrm{nS}$ to $\mathrm{G}=1.3 \mathrm{nS}$ for a charged $\mathrm{S}$ layer. $\mathrm{Cl}^{-}$conductance of the pore inverted from positive $(1.2 \mathrm{nS})$ in the neutral case, to negative $(-0.7 \mathrm{nS})$ in the charged case. Negative conductance of anions influx signifies that the charged nanopore is cation selective. $\mathrm{Na}^{+}$ conductance changed from $\mathrm{G}=1.2 \mathrm{nS}$ to $\mathrm{G}=-0.7 \mathrm{nS}$. Thus, the charged nanopore aids $\mathrm{NH}_{4}^{+}$acquisition, in striking contrast to the neutral nanopore, which inhibits nutrient influx.

\section{Ion concentrations and turnover are insensitive to model parameterization}

The short Debye lengths of the system contribute to a remarkable insensitivity of $\mathrm{NH}_{4}^{+}$concentrations to the 

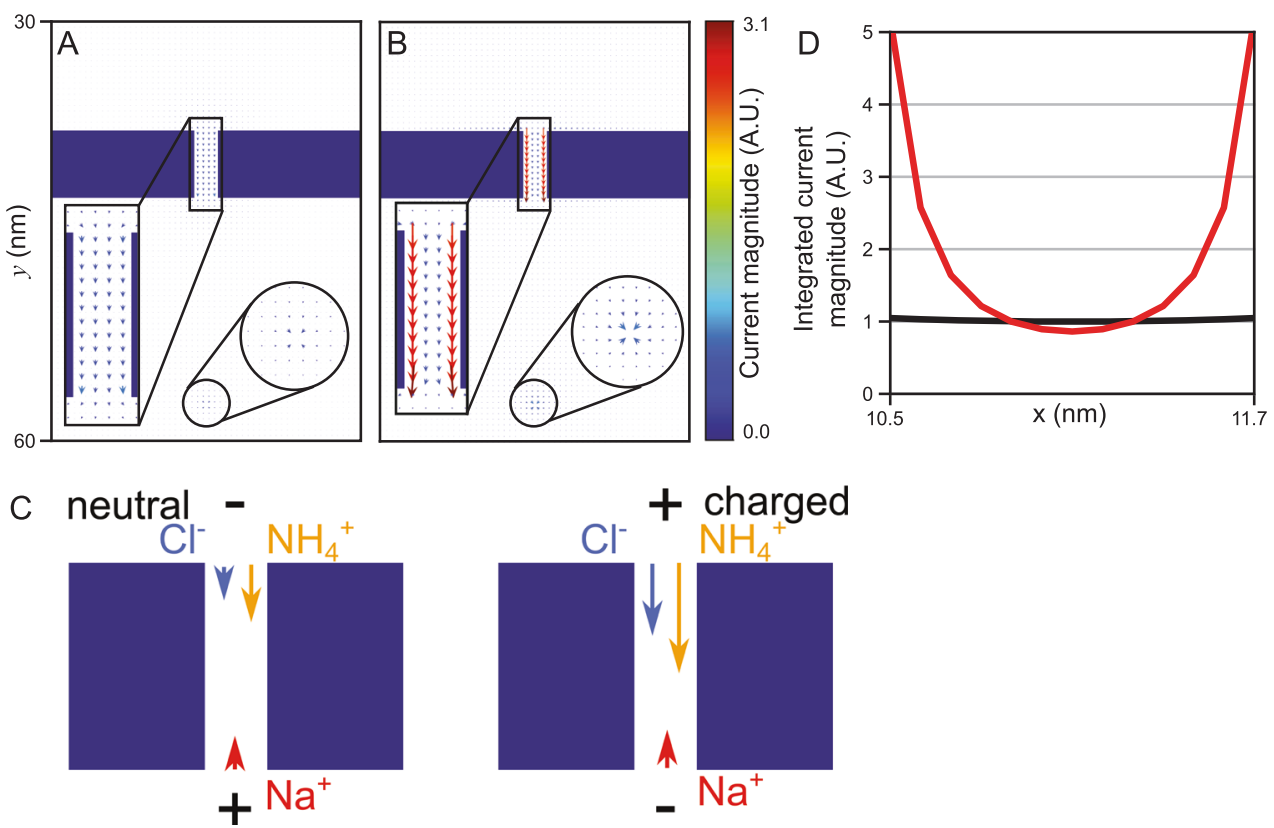

Fig. 5 Ion fluxes and currents at steady state predicted by the electrodiffusion reaction simulation. a $\mathrm{NH}_{4}^{+}$flux vector visualization in the bottom half of the simulation domain for a neutral S-layer at steady state. Both arrow length and color indicate the magnitude of flux at a particular point. Blue shaded areas indicate the S-layer. Magnitudes are normalized to the average value in the pore. $\mathbf{b ~} \mathrm{NH}_{4}{ }^{+}$flux vector visualization with a charged S-layer. Values normalized to panel (a). c Ion currents in the neutral (left) and charged (right) nanopore. Lengths of the arrows reflect the contribution to the total current of each ion species. The ' + ' and '-' signs indicate the polarity over the nanopore. d Magnitude of the vertical $\mathrm{NH}_{4}{ }^{+}$current component Jy across the pore width. A.U. arbitrary units geometric arrangement of the system's components. For example, the precise location of the AMO active site in PPS is unresolved. To investigate the effect of the active site distance from the S-layer nanopore in our simulations, we tested several different locations in PPS. We observed highly similar distributions of $\mathrm{NH}_{4}^{+}$for the charged S-layer for a wide range of positions (Fig. 6a). Our simulations suggested that the gain of a charged S-layer compared with a neutral S-layer is within $20 \%$, unless the active site is positioned very close to the S-layer nanopore (Fig. 6a, inset).

\section{Debye length scales of pore balance nutrient access and large particle sieving}

To examine the effect of the S-layer nanopore diameter on $\mathrm{NH}_{4}{ }^{+}$concentration at the active site, we carried out simulations with diameters ranging from 0.5 to $5.0 \mathrm{~nm}$ (Fig. 6b). The nanopore diameter affected the steady-state $\mathrm{NH}_{4}{ }^{+}$concentration at the active site in a nonlinear fashion. A nanopore diameter of $0.5 \mathrm{~nm}$ led to $\mathrm{NH}_{4}{ }^{+}$concentrations of $2.4 \mathrm{nM}$ (charged), and $0.7 \mathrm{nM}$ (neutral). For a $5 \mathrm{~nm}$ nanopore, in the case of a neutral S-layer the concentrations increased to $1.9 \mathrm{nM}$. The concentration increased modestly in the charged case, to $2.5 \mathrm{nM}$. The concentration at the active site for a charged S-layer remained remarkably constant over the range of nanopore diameters, to within $0.5 \%$. As the pore widens, the loss of Debye length overlap for the charged case could be offset by the increase in diameter. Interestingly, atomic models of experimentally determined SLPs suggest that nanopore sizes are in the $1.3-2.0 \mathrm{~nm}$ range [48, 49], precisely within the range of overlapping Debye lengths, and where the gain of a charged S-layer and nanopore is largest (Fig. 6b, inset). This signifies that the nanopore characteristic length scale could have evolved to balance its role as a size barrier to larger molecules while maintaining nutrient accessibility.

\section{Discussion}

Understanding the factors contributing to the ecological importance of AOA vs. AOB is critical for predicting their relative contributions to the biogeochemical cycling of nitrogen and carbon in aquatic, terrestrial, and engineered environments. An S-layer, instead of an outer membrane, is a key structural component that distinguishes AOA from AOB. Our simulations suggest that a charged, hexagonal S-layer can help concentrate cations essential for AOA metabolism in an EDL near the organism. We established 

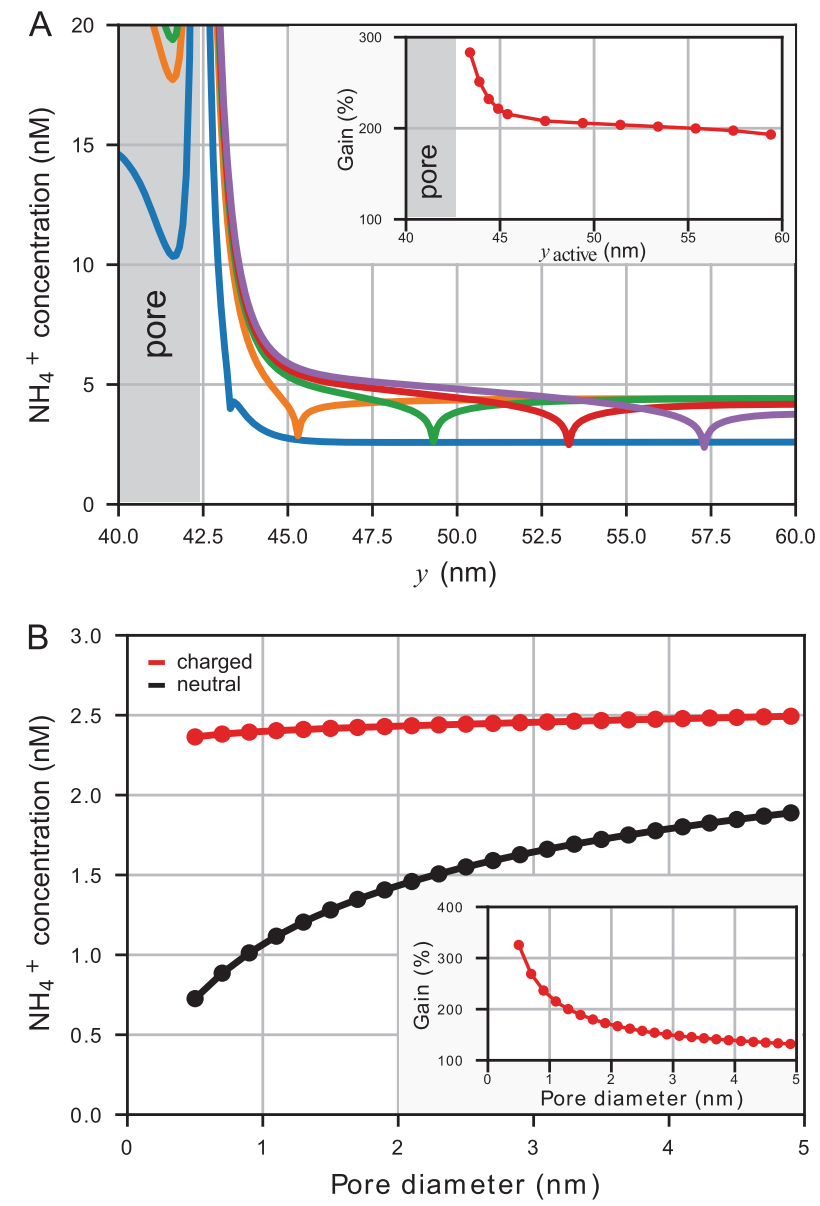

Fig. 6 Active site concentration insensitive to location or pore diameter. a $\mathrm{NH}_{4}{ }^{+}$concentration in PPS as a function of active site position (colored graphs), measured along the $x$-coordinate of the active site. For each graph, the active site is located at the cusp. Inset: the gain in $\mathrm{NH}_{4}^{+}$concentration at the active site for each position compared with a neutral S-layer. b $\mathrm{NH}_{4}{ }^{+}$concentration at the active site as a function of pore diameter for a charged (red) and neutral (black) S-layer. Inset: The percentage gain in $\mathrm{NH}_{4}{ }^{+}$concentration at the active site of a charged S-layer over a neutral S-layer as a function of pore size

that the characteristic length scale of the EDL, the Debye length, is less than a nanometer under marine $\mathrm{Na}^{+}$and $\mathrm{Cl}^{-}$ion concentrations, signifying that the AMO active site would not directly experience the effect of the nutrient reservoir. Unlike simulations of cell membrane potentials, our system does not assume a potential difference over the S-layer controlled by a selective pore. Instead, our results indicate that a symmetrically charged S-layer can expedite diffusion of charged nutrient solutes over the nanopore into the PPS when the electrodiffusion-reaction system is driven away from equilibrium, replenishing reacted $\mathrm{NH}_{4}{ }^{+}$in PPS. Under first-order Michaelis-Menten kinetics, this translated into a doubling of enzymatic turnover at low bulk concentration. This effect was largely insensitive to the geometry of our simulated system, such as pore diameter and placement of the AMO active site in the PPS. Removing the S-layer charge dramatically reduced ammonium concentration throughout the PPS. However, removing the S-layer altogether resulted in near-identical concentrations of $\mathrm{NH}_{4}^{+}$at the active site as the charged S-layer. Thus, the charge-effect appears to compensate for reduced nutrient access owing to the physical barrier of an S-layer.

Our results suggest that a charged S-layer is a functional adaptation that helps AOA compete in ecosystems with nutrient-limited conditions, such as the oligotrophic ocean where they greatly outnumber AOB. Nonetheless, the 'nutrient-harvesting' advantage resulting from a charged S-layer is insufficient to explain the gap in nutrient requirements between $\mathrm{AOA}$ and $\mathrm{AOB}$. Whereas a charged S-layer can elevate active site concentration and reaction turnover of AOA twofold, $\mathrm{AOB}$ require nutrient concentrations several orders of magnitude larger than AOA. Therefore, we speculate that AOA evolved additional cellular and molecular adaptations to sustain growth at $\mathrm{nM}$ concentrations.

Simulations of (synthetic) nanochannels commonly involve an externally applied potential difference that affects each, usually two, ion species equally [13]. By contrast, our biological model of a point-like $\mathrm{NH}_{4}{ }^{+}$sink creates an electrochemical gradient that acts asymmetrically. Although each species experienced an electric field owing to the sink, the chemical gradient acts directly on $\mathrm{NH}_{4}{ }^{+}$alone. This asymmetry resulted in sharply increased conductance, or ion transport number, for $\mathrm{NH}_{4}{ }^{+}$over the nanopore, whereas conductance of $\mathrm{Na}^{+}, \mathrm{Cl}-$, and $\mathrm{NH}_{4}{ }^{+}$ combined was relatively unaffected.

How does a charged, cation-selective nanopore aid substrate acquisition? Uncompensated negative charges in the PPS left behind by $\mathrm{NH}_{4}{ }^{+}$consumption could diffuse out through the nanopore. However, influx or efflux of $\mathrm{Cl}^{-}$is controlled by the cation-selective nanopore. We therefore propose the following mechanism of nanopore-facilitated $\mathrm{NH}_{4}{ }^{+}$transport in our model: An electrochemical gradient results in an influx of $\mathrm{NH}_{4}{ }^{+}$and $\mathrm{Na}^{+}$into the PPS. Efflux of $\mathrm{Cl}^{-}$is limited because the nanopore is cation selective, whereas $\mathrm{Na}^{+}$influx is balanced by its concentration gradient. Thus, a charge deficit owing to the sink is disproportionately compensated by influx of $\mathrm{NH}_{4}{ }^{+}$. How, then, would cation-selective nanopores transport nitrite, the metabolic end product of AOA [22], out of the PPS? Sufficiently high concentrations of $\mathrm{NO}_{2}{ }^{-}$in the PPS would eventually allow these ions to overcome a barrier electrical potential. We further speculate that high concentrations of negative charges in the PPS would raise the potential difference over the nanopore until an equilibrium is found, aiding $\mathrm{NH}_{4}^{+}$acquisition. 
Our simulations and other independent lines of evidence support that the substrate of AOA is ammonium $\left(\mathrm{NH}_{4}{ }^{+}\right)$, not ammonia $\left(\mathrm{NH}_{3}\right)$. In $\mathrm{AOB}$, intracellular access to $\mathrm{NH}_{3}$ is provided by both diffusion through lipid bilayers and through porins. Porins are conductive, membrane-bound channels that allow exchange of solutes [50] and are either non-specific or selective for anions or cations [51, 52]. Simulations have suggested that diffusion of ions through non-specific porins is driven by their concentration and charge gradients [53, 54]. Although S-layer pores, like porins, could facilitate concentration-driven diffusion of $\mathrm{NH} 3$, AOA lack a $\mathrm{NH}_{3}$-diffusive cell envelope, which would further reduce $\mathrm{NH}_{3}$ availability in the PPS. Additionally, at $\mathrm{pH}$ values encountered by $\mathrm{AOA}$, just $2 \%$ of 10 $\mathrm{nM}$ total ammonia concentration is in the unionized $\mathrm{NH}_{3}$ form. Taken together, we found that the concentration of $\mathrm{NH}_{3}$ at the active site, $24 \mathrm{pM}$, was orders of magnitude lower still than the $\mathrm{NH}_{4}{ }^{+}$concentration we found with a neutral S-layer (Fig S3). Finally, AOA (meta)genomes suggest that AMO has undergone significant adaptation compared with its bacterial counterpart, which could indicate a different molecular mechanism for ammonia oxidation. Nonetheless, we cannot firmly rule out $\mathrm{NH}_{3}$ as a substrate. For example, a controlled $\mathrm{pH}$ environment in the PPS would enable conversion from $\mathrm{NH}_{4}{ }^{+}$to $\mathrm{NH}_{3}$.

How can quantitative, predictive models like ours help resolve complex relationships in the microbial ecology of ammonia-oxidizing organisms? Environmental determinants underlying the distribution of ammonia-oxidizing populations remain poorly understood. For example, the ratios of $\mathrm{AOA}$ to $\mathrm{AOB}$ in estuarine sediment were found to generally decline along an increasing salinity gradient [55]. Although availability of sedimentary $\mathrm{NH}_{4}{ }^{+}$is known to decline with increasing ionic strength [56], and $\mathrm{NH} 3$ availability should be relatively unaffected, the population shifts defied a simple relationship [55]. Instead, such population shifts likely involve a complex, multidimensional response on cellular and molecular scales. Our model suggests that transport properties of AOA nanopores can counteract declining substrate availability at increasing ionic strength owing to more favorable Debye length scales, blurring apparent ammonia oxidizer ecological niches. Additionally, the evolutionary gain of a charged S-layer for acquiring $\mathrm{NH}_{4}^{+}$in oligotrophic systems highlights adaptations that have made AOA more abundant in the (deep) open ocean, where $\mathrm{NH}_{4}{ }^{+}$concentrations are low.

Several improvements to our model could further elucidate if the remarkable efficiency of AOA is rooted in cellular or molecular adaptations, or both. Besides $\mathrm{NO}_{2}{ }^{-}$[22], AMO activity will almost certainly introduce reactive intermediates [57] into the PPS, which are currently unaccounted for in our model. For example, nitric oxide (NO) is a key intermediate [58], which could aid in the formation of
$\mathrm{NO}_{2}{ }^{-}$through the oxidation of hydroxylamine [57, 59]. Additionally, NO can react abiotically to form $\mathrm{N}_{2} \mathrm{O}$ [57]; both of which could diffuse out of the cell and lead to reduction in total $\mathrm{NO}_{2}{ }^{-}$production. It is important to note that all known AOA encode a nitrite reductase (NirK) but have no nitric oxide reductase (e.g., [31]), and thus are not capable of true nitrifier denitrification. Total mass and charge are not conserved in the simulation, which limits the time-scale for which our results are valid. Indeed, the persistent currents carried by $\mathrm{Na}^{+}$and $\mathrm{Cl}^{-}$may be partially artefactual. Structural models of SLPs, AMO, and other, related proteins of the AOA ammonia oxidation pathway can also help inform the biophysical parameters of our simulation. For example, the location and identities of amino acids lining the nanopore are critically important to accurately model the electric field. Structures and integrated modeling can also help us estimate kinetics by molecular dynamics simulation [60]. Simulating ions using finite size will also improve our model.

Such improvements can help test theories about the ammonia oxidation pathway in archaea. For example, comparative analyses of AOA genomes revealed a pair of highly conserved ammonia transporters across ecotypes [61]. Although ammonia is often a nitrogen source for amino-acid synthesis, facilitated transport across the inner membrane also raises the possibility of the cytoplasm as an ammonia reservoir for AMO. Accordingly, a recent hypothesis ascribed the extreme efficiency of AOA to cellular uptake of $\mathrm{NH}_{4}{ }^{+}$by ammonia transporters, followed by diffusion of membrane-permeative $\mathrm{NH}_{3}$ into the PPS [29]. However, this model would seem contrary to ammonia toxicity observed in non-terrestrial AOA at low millimolar concentrations [22].

Organisms with S-layers inhabit virtually every environment on the planet, including extreme conditions experienced by AOA and many other archaea. Analysis of characterized S-layer amino-acid sequences indicates that most SLPs are positively or negatively charged. Through our simulations, we have found that charged S-layers and nanopores enhance diffusion of charged solutes irrespective of parameterization. Notably, a neutral S-layer inhibits diffusion of charged molecules, indicating a potential fitness disadvantage of neutral cell envelope components. Thus, S-layer charge may have emerged by convergent evolution to enhance metabolic function in diverse ecosystems.

\section{Materials and methods}

\section{Zeta potential}

We evaluated the charge density model we derived above by measuring the Zeta potential. $N$. limnia strain SFB1 was 
grown in modified SCM25H media (pH 7.5, 8 salinity) containing HEPES ( $1 \mathrm{M}, \mathrm{pH} 7.5)$ and $100 \mu \mathrm{M}$ ammonium chloride [39]. N. limnia SFB1 is an enrichment culture containing $>97 \%$ SFB1 cells that has been maintained through monthly serial transfers into fresh $\mathrm{SCM} 25 \mathrm{H}$ media $\left(10 \%\right.$ inoculum) at room temperature $\left(20-22{ }^{\circ} \mathrm{C}\right)$. Samples were taken during early stationary phase, $\mathrm{pH}$ adjusted with $\mathrm{HCl}$ or $\mathrm{NaOH}$ (depending on target $\mathrm{pH}$ ), and immediately measured for Zeta potential using a Zetasizer Nano ZS (Malvern Instruments Ltd). The measurement was performed with $0.14 \mathrm{M}$ artificial seawater, which has a Debye length of $8.25 \AA$, and revealed a Zeta potential of $-7 \mathrm{mV}$ at $\mathrm{pH}$ 7. Then, we performed analytical calculation with the same setting, that is, $0.14 \mathrm{M}$ salt water and charge density $0.02 \mathrm{e}^{-} / \AA$, and found that $-7 \mathrm{mV}$ potential was located at a distance of $18 \AA$ from the charged S-layer surface.

\section{Cryoelectron tomograms}

We imaged the S-layer of $N$. maritimus with electron cryotomography (ECT). We concentrated growing culture by filtration. The concentrated cells were suspended in phosphate-buffered saline containing bovine serum albumin-treated colloidal gold fiducial markers $(10 \mathrm{~nm})$ $[62,63]$. Grids were prepared and plunge frozen with a Vitrobot Mark III ((FEI Company, Hillsboro, OR) maintained at $80 \%$ humidity and $30^{\circ} \mathrm{C}$ temperature. Three $\hat{A}_{\mu l}$ culture solution was applied to R2/2 copper Quantifoil EM grids (Quantifoil Micro Tools, Großlöbichau, Germany), the excess liquid was blotted and the grids were plunged frozen into a liquid ethane-propane mixture. The grids were imaged with a FEI PolaraTM (FEI Company, Hillsboro, OR) $300 \mathrm{kV}$ field emission gun transmission electron microscope. The tomograms were collected at 22,000 $\tilde{A}$-magnifications and were analyzed with the UCSF Tomo (University of California, San Francisco, CA) and the IMOD software packages. Subtomogram averaging was performed using particle estimation for electron tomography (PEET) in IMOD [64], which resulted in images of the subunits at $4 \mathrm{~nm}$ resolution.

\section{PNP electrodiffusion reaction model}

Researchers previously established that ionic transport through charged nanopores is dominated by electrophoretic ion migration [35, 37]. Convection and diffusion were found to contribute marginally, justifying our choice of a PNP model. The Nernst-Planck (NP) equation describes the diffusion of charged particles under an ionic concentration gradient $\nabla c$ and an electric field $E=-\nabla \phi$. We added a reaction term to the $\mathrm{NP}$ equation, representing ion consumption proportional to the local concentration:

$$
\begin{gathered}
\frac{\partial c}{\partial t}=-\nabla \cdot J+R(c)=-\nabla \cdot\left[-D \nabla c+\frac{N_{A} e D z}{R T} c E\right] \\
-\frac{k}{k_{M}} c N_{A} \delta\left(x-x_{\mathrm{amo}}\right),
\end{gathered}
$$

\begin{tabular}{|c|c|c|c|}
\hline$J$ & flux & $E$ & electric field $[\mathrm{V} / \mathrm{m}]$ \\
\hline$R(c)$ & reactive term & $R$ & gas constant \\
\hline$D$ & $\begin{array}{l}\text { diffusion coefficient of the } \\
\text { chemical species }\end{array}$ & $T$ & temperature $[\mathrm{K}]$ \\
\hline$c$ & concentration $\left[\mathrm{M} / \mathrm{m}^{3}\right]$ & $k$ & $\begin{array}{l}\text { enzymatic turnover } \\
\text { rate }\left[\mathrm{s}^{-1}\right]\end{array}$ \\
\hline$N_{\mathrm{A}}$ & Avogadro's constant & $k_{\mathrm{m}}$ & $\begin{array}{l}\text { Michaelis constant } \\
{[\mathrm{M}]}\end{array}$ \\
\hline$e$ & elementary charge $[\mathrm{C}]$ & $\delta$ & Dirac delta function \\
\hline$z$ & valence & $x_{\mathrm{am}}$ & $\begin{array}{l}\text { position of the } \\
\text { AMO active site }\end{array}$ \\
\hline
\end{tabular}

where

In our simulations, $\quad T=300 \mathrm{~K}, k=10 \mathrm{~s}^{-1}$. Conventionally, the Poisson equation, which is derived from the charge distribution, is solved to obtain the electrostatic potential

$-\nabla \cdot(\varepsilon \nabla \phi)=4 \pi \rho_{f}+4 \pi z c$,

where

$\rho_{f}(x)=\sum_{j} z_{j} \delta\left(x-x_{j}\right)$

represents the fixed charge distribution, and $4 \pi z c$ represents the mobile ions. To improve computational performance, we used an alternative, equivalent implementation, which replaces the Poisson equation by the current equation

$\frac{\partial E}{\partial t}=-\frac{N_{A} e}{\varepsilon} \sum_{i=1}^{r} z_{i} J_{i}$

Finally, the flux of each ion is updated by

$J=D[\nabla c] \frac{e N_{A}}{T} D z c E$.

The coupled system (1) and (2) is known as the PNP model [65-67].

\section{PNP simulation}

We implemented a finite-difference time-domain solver for the PNP equations. The simulation was performed on a 
$22 \times 60 \mathrm{~nm}$ simulation domain with periodic boundaries along the longest dimension, with grid spacing $0.1 \mathrm{~nm}$ giving $n_{\mathrm{x}}=221$ by $n_{\mathrm{y}}=601$ elements. $\mathrm{NH}_{4}{ }^{+}$ions were added at concentrations of 10,100 , and $100 \mathrm{nM}$. Sodium $\left(\mathrm{Na}^{+}\right)$co-ions and chloride $\left(\mathrm{Cl}^{-}\right)$counterions were added at $0.14 \mathrm{M}$ concentration to model a marine ecosystem. We ensured electroneutrality for the three ion species at the bulk boundary. An integration step size of 1 picosecond was selected (Fig S1). All simulations were carried out for at least 10 microseconds. Selected simulations were run for up to 100 microseconds to study behavior of the current at steady state. At each time step, eleven profiles in 2D space were updated and stored: the concentrations of ammonium $\mathrm{NH}_{4}{ }^{+}, \mathrm{Na}^{+}$, and $\mathrm{Cl}^{-}$, the fluxes $J_{\mathrm{x}, \mathrm{y}}$ of the three ion species, and electric fields $E_{\mathrm{x}, \mathrm{y}}$. An ion sink, with a turnover rate $k=$ $10 \mathrm{~s}^{-1}$ was placed $2.5 \mathrm{~nm}$ from the bottom lipid membrane (15.5 nm from the S-layer's lower pore mouth) to represent the ammonia oxidation enzyme, that is, AMO. Simulations were carried out with Matlab R2016b (MathWorks, Natick, MA) and custom $\mathrm{C}++$ code. The code is available from https://github.com/ExcitedStates.

\section{Boundary conditions}

We implemented periodic boundaries at $x=0 \mathrm{~nm}$ and $x=22 \mathrm{~nm}$, that is, $c\left(x_{1=0}, y\right)=c\left(x_{1=\mathrm{W}}, y\right)$ and $c\left(x_{1=\mathrm{W}+1}, y\right)$ $=c\left(x_{1=1}\right)$, where $l=0, \ldots, W$ denotes the index of the cells along the $x$ direction. To model impermeability of the Slayer and the cytoplasmic membrane to charged ion species, we applied a Neumann boundary condition that prohibits flux in a direction normal to these surfaces: $J_{\mathrm{i}, \mathrm{u}}=0$ for all ion species $i$, where $u$ is the normal direction of the S-layer boundary or cell membrane. We implemented a diffusive boundary condition [68] at the top boundary, such that the inward flux of each ion is proportional to the difference of concentration between the top boundary of the domain and the bulk concentration, that is,

$J_{i, y}^{(t)}(x, y=0.5)=\frac{D}{\Delta x}\left[c_{i}^{(t)}(x, y=1)-c_{i, \text { bulk }}\right]$,

with $y=0.5$ indicating the position of the top boundary of the simulation domain, that is, immediately above the top-most cells (Suppl. Fig. S5). The choice of $c_{i \text {,bulk }}$ is stated below. We observed that diffusive boundary conditions were sufficient to replenish the ions consumed at the sink.

\section{Initial conditions}

We calculated initial concentration profiles $c_{i}^{(t=0)}$, from analytical solutions to the Poisson-Boltzmann equations for a charged surface in the absence of a pore. In this case, the initial conditions are simply a function of the distance $d$ from the S-layer. We calculated the Debye length $l_{\mathrm{D}}=\kappa^{-1}$ and the Gouy-Chapman length $l_{\mathrm{GC}}$ given the surface charge density $0.02 \mathrm{e}^{-} / \AA \mathrm{As}^{2}$ and a bulk concentration of salt $c_{\text {salt } \pm, b u l k}$ of $0.14 \mathrm{M}$ as $l_{D}=\sqrt{\frac{\varepsilon k_{B} T}{8 \pi e^{2} n_{0}}}$, and $l_{G C}=\frac{\varepsilon T}{2 \pi e|\sigma|}, k_{\mathrm{B}}$ denotes the Boltzmann constant, and $c_{0}$ is the bulk concentration in moles per $m^{3}$. The resulting potential at distance $d$ from the S-layer is $\quad \psi(d)=-\frac{2 T}{e} \ln \frac{1+\gamma \exp -d / l_{D}}{1-\gamma \exp -d / l_{D}}, \quad$ where $\quad \gamma=$ $-\frac{l_{G C}}{\lambda_{D}}+\sqrt{\left(\frac{l_{G C}}{\lambda_{D}}\right)^{2}+1}$ is the positive root of a quadratic equation, and the concentrations of $\mathrm{Na}^{+}$and $\mathrm{Cl}^{-}$are given by $c_{\text {salt }}(d)=c_{\text {salt }}^{(t=0)}(d)=\left(\frac{1 \pm \gamma \exp -d / l_{D}}{1 \mp \gamma \exp -d / l_{D}}\right)^{2}$. The initial concentration of ammonium is derived by rescaling the concentration of $\mathrm{Na}^{+}$, that is, $c_{\mathrm{NH}_{4}^{+}}^{(t=0)}=\frac{c_{\mathrm{NH}_{4}^{+}, \text {bulk }}}{c_{\mathrm{Na}^{+}, \text {bulk }}} c_{\mathrm{Na}^{+}}^{(t=0)}$. PNP simulations were started from these initial profiles to obtain the steady state with charges and reacting enzyme turned on. For simulations with a neutral S-layer, we assumed uniform initial concentration profiles in the absence of electric fields.

\section{Surface charge density}

The structure of AOA SLPs is unknown. To obtain an estimate for the surface charge density of the AOA Slayer, we therefore used the published structure of the $M$. acetivorans SLP as a guide [48]. First, we classified each residue of the SLP gene Nmar1201 as either exposed or buried [69]. The prediction revealed that residues in the protein are, on average, $78.7 \%$ exposed. Asp and Glu make up $26.2 \%$ of the amino-acid composition of Nmar1201, and are, on average, $85 \%$ exposed. We further examined the surface charge density as a function of $\mathrm{pH}$. We assumed that each exposed acidic residue has negative unit charge if the $\mathrm{pH}$ is much larger than its $\mathrm{pK}_{\mathrm{A}}$, neutral if the $\mathrm{pH}$ is much smaller than $\mathrm{pK}_{\mathrm{A}}$, or minus 0.5 -charged when $\mathrm{pH}=\mathrm{pK}_{\mathrm{A}}$. Exposed basic residues are treated similarly. This model suggested that increasing $\mathrm{pH}$ will reduce S-layer charge. We then counted the number of charged residues in Nmar1201 at $\mathrm{pH}=7$, obtaining a net charge of $210 \mathrm{e}^{-}$. We assumed, conservatively, that each asymmetric unit in an S-layer hexagonal repeating tile is a monomer, so that each tile consists of nine SLPs (Suppl. Fig. S5). Based on the dimensions derived from the tomogram (Fig. 1), the estimated surface area of each hexagonal tile is $110^{2} \pi \mathrm{nm}^{2}$ on each side (top and bottom). Accordingly, we estimated a surface charge density of $0.02 \mathrm{e}^{-} / \AA^{2}$. 


\section{Enzyme turnover}

We estimated the enzymatic turnover of $N$. maritimus SCM1 from batch cultures ([22], Fig. 1). The amount of ammonia in a $1 \mathrm{~L}$ culture is 100 millimoles. The number of AOA cells in a $1 \mathrm{~L}$ culture is approximately $10^{9}$, and each cell has approximately $10^{4}$ AMOs. The ammonia was consumed in ca. 10 days. Therefore, a single AMO active site has a turnover of 6.94 per second, which we rounded to 10 per second.

\section{Electrical properties}

We calculated the potential across the pore by integrating $E_{\mathrm{y}}$ with respect to $y$ over the depth of the pore $([37.9,42.5] \mathrm{nm})$ and averaging over the width of the pore. The electrical current is calculated by $I=z J_{\mathrm{y}, \text { avg }} N_{A} e(\Delta x)^{2}$, where $J_{\mathrm{y} \text {,avg }}$ is obtained by integrating $J_{\mathrm{y}}$ with respect to $x$ and averaging over the depth of the pore $([10.5,11.7] \mathrm{nm})$. The sign of $G=I / V$ follows the convention that flux of negative charge toward positive potential $\mathrm{V}$ is positive conductance.

Acknowledgements This work was partially supported by the US Department of Energy, Laboratory Directed Research and Development under contract No. DE-AC02-76SF00515. JH was supported by the National Science Foundation Graduate Research Fellowship Program (NSF-GRFP), as well as the US Department of Energy Office of Science Graduate Student Research Program (DOE-SCGSR). FP acknowledges support from the National Institutes of Health (NIH), grant No. R35GM122543. DAS was funded in part by the United States National Science Foundation Grants MCB-092074 and OCE1046017. HvdB acknowledges support from the U.S. Department of Energy, Office of Science, Office of Advanced Scientific Computing Research, Scientific Discovery through Advanced Computing (SciDAC) program. Computations were performed at the Stanford Research Computing Center. Glycosylation analysis by mass spectrometry was possible with assistance from C Adams and R Lieb (Stanford University Mass Spectrometry).

\section{Compliance with ethical standards}

Conflict of interest The authors declare that they have no conflict of interest.

\section{References}

1. Sleytr UB, Sára M. Bacterial and archaeal s-layer proteins: structure-function relationships and their biotechnological applications. Trends Biotechnol. 1997;15:20-6.

2. Albers SV, Meyer BH. The archaeal cell envelope. Nat Rev Microbiol. 2011;9:414-26.

3. Madhurantakam C, Howorka S, Remaut H. S-layer structure in bacteria and archaea. In: Barton L., Bazylinski D., Xu H. (eds) Nanomicrobiology. Springer, NY, New York, 2014. p. 11-37.

4. Fagan RP, Fairweather NF. Biogenesis and functions of bacterial S-layers. Nat Rev Microbiol. 2014;12:211-22.

5. McDougall M, Francisco O, Viddal C, Roshko R, Meier M, Stetefeld J. Archaea S-layer nanotube from a "black smoker" in complex with cyclo-octasulfur $\mathrm{S}_{8}$ rings. Proteins. 2017;85: 2209-16.

6. Gerbino E, Carasi P, Mobili P, Serradell MA, Gómez-Zavaglia A. Role of S-layer proteins in bacteria. World J Microbiol Biotechnol. 2015;31:1877-87.

7. König H. Archaeobacterial cell envelopes. Can J Microbiol. 1988;34:395-406.

8. Engelhardt H. Mechanism of osmoprotection by archaeal s-layers: a theoretical study. J Struct Biol. 2007;160:190-9.

9. Kish A, Miot J, Lombard C, Guigner JM, Bernard S, Zirah S, et al. Preservation of archaeal surface layer structure during mineralization. Sci Rep. 2016;6:26152.

10. Sára M, Sleytr UB. Molecular sieving through S-layers of bacillus stearothermophilus strains. J Bacteriol. 1987;169:4092-8.

11. Nikaido H, Vaara M. Molecular basis of bacterial outer membrane permeability. Microbiol Rev. 1985;49:1-32.

12. Sotiropoulou S, Mark SS, Angert ER, Batt CA. Nanoporous Slayer protein lattices. A biological ion gate with calcium selectivity. J Phys Chem C. 2007;111:13232-7.

13. Tagliazucchi M, Szleifer I. Transport mechanisms in nanopores and nanochannels: can we mimic nature? Mater Today. 2015; 18:131-42.

14. Valentine DL. Adaptations to energy stress dictate the ecology and evolution of the archaea. Nat Rev Microbiol. 2007;5: 316-23.

15. Brochier-Armanet C, Boussau B, Gribaldo S, Forterre P. Mesophilic crenarchaeota: proposal for a third archaeal phylum, the thaumarchaeota. Nat Rev Microbiol. 2008;6:245-52.

16. Leininger S, Urich T, Schloter M, Schwark L, Qi J, Nicol GW, et al. Archaea predominate among ammonia-oxidizing prokaryotes in soils. Nature. 2006;442:806-9.

17. Wuchter C, Abbas B, Coolen MJL, Herfort L, Bleijswijk J, van Timmers P, et al. Archaeal nitrification in the ocean. Proc Natl Acad Sci USA. 2006;103:12317-22.

18. Michael Beman J, Popp BN, Francis CA. Molecular and biogeochemical evidence for ammonia oxidation by marine crenarchaeota in the gulf of california. ISME J. 2008;2:429-41.

19. Stahl DA, Torre JRdela. Physiology and diversity of ammoniaoxidizing archaea. Annu Rev Microbiol. 2012;66:83-101.

20. Prosser JI, Nicol GW. Relative contributions of archaea and bacteria to aerobic ammonia oxidation in the environment. Environ Microbiol. 2008;10:2931-41.

21. Santoro AE, Casciotti KL, Francis CA. Activity, abundance and diversity of nitrifying archaea and bacteria in the central california current. Environ Microbiol. 2010;12:1989-2006.

22. Martens-Habbena W, Berube PM, Urakawa H, Torre JRdela, Stahl DA. Ammonia oxidation kinetics determine niche separation of nitrifying archaea and bacteria. Nature. 2009;461: 976-9.

23. Horak REA, Qin W, Schauer AJ, Armbrust EV, Ingalls AE, Moffett JW, et al. Ammonia oxidation kinetics and temperature sensitivity of a natural marine community dominated by archaea. ISME J. 2013;7:2023-33.

24. Park BJ, Park SJ, Yoon DN, Schouten S, Sinninghe Damste JS, Rhee SK. Cultivation of autotrophic ammonia-oxidizing archaea from marine sediments in coculture with sulfur-oxidizing bacteria. Appl Environ Microbiol. 2010;76:7575-87.

25. Sato C, Schnoor JL, McDonald DB, Huey J. Test medium for the growth of nitrosomonas europaea. Appl Environ Microbiol. 1985;49:1101-7.

26. Ward BB. Kinetic studies on ammonia and methane oxidation by Nitrosococcus oceanus. Arch Microbiol. 1987;147:126-33.

27. Sayavedra-Soto L, Arp D. Ammonia-oxidizing bacteria: their biochemistry and molecular biology. In: Ward B, Arp D, Klotz M, (eds). Nitrification. ASM Press, DC, Washington, 2011. p. 11-37. 
28. Kerou M, Offre P, Valledor L, Abby SS, Melcher M, Nagler M, et al. Proteomics and comparative genomics of Nitrososphaera viennensis reveal the core genome and adaptations of archaeal ammonia oxidizers. Proc Natl Acad Sci USA. 2016;113: E7937-46.

29. Lehtovirta-Morley LE, Sayavedra-Soto LA, Gallois N, Schouten S, Stein LY, Prosser JI, et al. Identifying potential mechanisms enabling acidophily in the ammonia-oxidizing archaeon "Candidatus Nitrosotalea devanaterra”. Appl Environ Microbiol. 2016; 82:2608-19.

30. Tolar BB, Herrmann J, Bargar JR, van den Bedem H, Wakatsuki $\mathrm{S}$, Francis CA. Integrated structural biology and molecular ecology of N-cycling enzymes from ammonia-oxidizing archaea. Environ Microbiol Rep. 2017;9:484-91.

31. Walker CB, Torre JR, de la, Klotz MG, Urakawa H, Pinel N, Arp DJ, et al. Nitrosopumilus maritimus genome reveals unique mechanisms for nitrification and autotrophy in globally distributed marine crenarchaea. Proc Natl Acad Sci USA. 2010;107: 8818-23.

32. Suzuki I, Dular U, Kwok SC. Ammonia or ammonium ion as substrate for oxidation by Nitrosomonas europaea cells and extracts. J Bacteriol. 1974;120:556-8.

33. Hatzenpichler R. Diversity, physiology, and niche differentiation of ammonia-oxidizing archaea. Appl Environ Microbiol. 2012;78: 7501-10.

34. Gorman-Lewis D, Martens-Habbena W, Stahl DA. Thermodynamic characterization of proton-ionizable functional groups on the cell surfaces of ammonia-oxidizing bacteria and archaea. Geobiology. 2014;12:157-71.

35. Daiguji H, Yang P, Majumdar A. Ion transport in nanofluidic channels. Nano Lett. 2004;4:137-42.

36. Stein D, Kruithof M, Dekker C. Surface-charge-governed ion transport in nanofluidic channels. Phys Rev Lett. 2004;93:035901.

37. Ho C, Qiao R, Heng JB, Chatterjee A, Timp RJ, Aluru NR, et al. Electrolytic transport through a synthetic nanometer-diameter pore. Proc Natl Acad Sci USA. 2005;102:10445-50.

38. Duan C, Majumdar A. Anomalous ion transport in 2-nm hydrophilic nano channels. Nat Nanotechnol. 2010;5:848-52.

39. Mosier AC, Lund MB, Francis CA. Ecophysiology of an ammonia-oxidizing archaeon adapted to low-salinity habitats. Microb Ecol. 2012;64:955-63.

40. Herrmann J, Jabbarpour F, Bargar PG, Nomellini JF, Li PN, Lane $\mathrm{TJ}$, et al. Environmental calcium controls alternate physical states of the caulobacter surface layer. Biophys J. 2017;112: 1841-51.

41. Calo D, Kaminski L, Eichler J. Protein glycosylation in archaea: sweet and extreme. Glycobiology. 2010;20:1065-76.

42. Peyfoon, E, Meyer, B, Hitchen, PG, Panico, M, Morris, HR, Haslam, SM, et al. The S-layer glycoprotein of the crenarchaeote Sulfolobus acidocaldarius is glycosylated at multiple sites with chitobiose-linked N-glycans. Archaea 2010.

43. Baumeister W, Wildhaber I, Phipps BM. Principles of organization in eubacterial and archaebacterial surface proteins. Can J Microbiol. 1989;35:215-27.

44. Qin W, Amin SA, Lundeen RA, Heal KR, Martens-Habbena W, Turkarslan S, et al. Stress response of a marine ammoniaoxidizing archaeon informs physiological status of environmental populations. ISME J. 2018;12:508-19.

45. Krogh A, Larsson B, Heijne Gvon, Sonnhammer EL. Predicting transmembrane protein topology with a hidden markov model: application to complete genomes. J Mol Biol. 2001;305: 567-80.

46. Yachdav G, Kloppmann E, Kajan L, Hecht M, Goldberg T, Hamp $\mathrm{T}$, et al. PredictProtein-an open resource for online prediction of protein structural and functional features. Nucleic Acids Res. 2014;42:W337-43.
47. Eichler J, Maupin-Furlow J. Post-translation modification in archaea: lessons from haloferax volcanii and other haloarchaea. FEMS Microbiol Rev. 2013;37:583-606.

48. Arbing MA, Chan S, Shin A, Phan T, Ahn CJ, Rohlin L, et al. Structure of the surface layer of the methanogenic archaean methanosarcina acetivorans. Proc Natl Acad Sci USA. 2012;109:11812-7.

49. Bharat TAM, Kureisaite-Ciziene D, Hardy GG, Yu EW, Devant JM, Hagen WJH, et al. Structure of the hexagonal surface layer on caulobacter crescentus cells. Nat Microbiol. 2017;2:17059.

50. Galdiero S, Falanga A, Cantisani M, Tarallo R, Della Pepa ME, D'Oriano V, et al. Microbe-host interactions: structure and role of gram-negative bacterial porins. Curr Protein Pept Sci. 2012;13:843-54.

51. Benz R, Schmid A, Hancock RE. Ion selectivity of gram-negative bacterial porins. J Bacterid. 1985;162:722-7.

52. Ritchie RJ. The ammonia transport, retention and futile cycling problem in cyanobacteria. Microb Ecol. 2013;65:180-96.

53. Im W, Roux B. Ion permeation and selectivity of OmpF porin: a theoretical study based on molecular dynamics, brownian dynamics, and continuum electrodiffusion theory. J Mol Biol. 2002a;322:851-69.

54. Im W, Roux B. Ions and counterions in a biological channel: a molecular dynamics simulation of OmpF porin from Escherichia coli in an explicit membrane with $1 \mathrm{M} \mathrm{KCl}$ aqueous salt solution. J Mol Biol. 2002b;319:1177-97.

55. Bernhard AE, Landry ZC, Blevins A, Torre JR, de la, Giblin AE, Stahl DA. Abundance of ammonia-oxidizing archaea and bacteria along an estuarine salinity gradient in relation to potential nitrification rates. Appl Environ Microbiol. 2010;76:1285-9.

56. Rysgaard S, Thastum P, Dalsgaard T, Christensen PB, Sloth NP. Effects of salinity on $\mathrm{NH}_{4}+$ adsorption capacity, nitrification, and denitrification in Danish estuarine sediments. Estuaries. 1999;22:21-30.

57. Kozlowski JA, Stieglmeier M, Schleper C, Klotz MG, Stein LY. Pathways and key intermediates required for obligate aerobic ammonia-dependent chemolithotrophy in bacteria and thaumarchaeota. ISME J. 2016;10:1836-45.

58. Martens-Habbena W, Qin W, Horak REA, Urakawa H, Schauer AJ, Moffett JW, et al. The production of nitric oxide by marine ammonia-oxidizing archaea and inhibition of archaeal ammonia oxidation by a nitric oxide scavenger. Environ Microbiol. 2015; 17:2261-74.

59. Stieglmeier M, Mooshammer M, Kitzler B, Wanek W, Zechmeister-Boltenstern S, Richter A, et al. Aerobic nitrous oxide production through $\mathrm{N}$-nitrosating hybrid formation in ammoniaoxidizing archaea. ISME J. 2014;8:1135-46.

60. van den Bedem H, Fraser JS. Integrative, dynamic structural biology at atomic resolution - it's about time. Nat Methods. 2015;12:307-18.

61. Offre P, Kerou M, Spang A, Schleper C. Variability of the transporter gene complement in ammonia-oxidizing archaea. Trends Microbiol. 2014;22:665-75.

62. Iancu CV, Tivol WF, Schooler JB, Dias DP, Henderson GP, Murphy GE, et al. Electron cryotomography sample preparation using the vitrobot. Nat Protoc. 2006;1:2813-9.

63. Mastronarde, DN. Fiducial marker and hybrid alignment methods for single- and double-axis tomography. In: Frank J. (ed) Electron tomography. Springer, NY, New York, 2007. p. 163-85.

64. Nicastro D, Schwartz C, Pierson J, Gaudette R, Porter ME, McIntosh JR. The molecular architecture of axonemes revealed by cryoelectron tomography. Science. 2006;313:944-8.

65. Cohen H, Cooley JW. The numerical solution of the timedependent Nernst-Planck equations. Biophys J. 1965;5:145-62.

66. Brumleve TR, Buck RP. Numerical solution of the Nernst-Planck and poisson equation system with applications to membrane 
electrochemistry and solid state physics. J Electroanal Chem Interfacial Electrochem. 1978;90:1-31.

67. Lu B, Holst MJ, McCammon JA, Zhou YC. Cultivation of autotrophic ammonia-oxidizing archaea from marine sediments in coculture with sulfur-oxidizing bacteria. Appl Environ Microbiol. 2010;76:7575-87.
68. Lelidis I, Ross Macdonald J, Barbero G. Poisson-Nernst-Planck model with Chang-Jaffe, diffusion, and ohmic boundary conditions. J Phys D Appl Phys. 2015;49:025503.

69. Song J, Tan H, Mahmood K, Law RHP, Buckle AM, Webb GI, et al. Prodepth: predict residue depth by support vector regression approach from protein sequences only. PLoS One. 2009;4:e7072. 